\title{
Metodología cualitativa como herramienta en la investigación de la calidad de vida
}

\section{Lilia Janeth Torres-Chávez ${ }^{1}$}

\section{Aporte de estudiante}

Posgrado

Resumen: La calidad de vida es un constructo complejo que se ha desarrollado a partir de los años sesenta, se ha definido a través de indicadores objetivos y medibles, así como de indicadores subjetivos, o bien, de la combinación de ambos, así como se han dado a conocer distintos modelos teóricos para explicarla, sin embargo, se plantea la situación de que generalmente la calidad de vida se valora a través de cuestionarios psicométricos desde un enfoque metodológico cuantitativo. La metodología cualitativa ha sido demeritada en el pasado como parte de la investigación científica, sin embargo, actualmente es considerada como una metodología válida, holística e inductiva, se trata de un proceso interactivo para comprender diversos fenómenos y significados a profundidad con la posibilidad de generar teoría. Este ensayo considera de manera breve, las circunstancias para valorar la calidad de vida a través de un enfoque metodológico cualitativo. El desarrollo abarca elementos generales de la calidad de vida, así como elementos generales de la metodología cualitativa con el objetivo de conocer y establecer una relación en la que se valore la efectividad para estudiar la calidad de vida a través de métodos cualitativos, considerando factores importantes como el contexto, el tiempo y las personas.

\section{Introducción}

La calidad de vida (CV) se puede definir como "la percepción que cada persona tiene de su propio lugar en la vida, dentro de un contexto cultural y del sistema de valores al que se conforma, en relación con las expectativas, intereses y logros" (Tonon, 2015, p. 5). El término calidad de vida, así como las formas de evaluarla se han desarrollado y modificado a lo largo del tiempo, de acuerdo a la época, el lugar o las personas. Generalmente abordamos la calidad de vida desde una perspectiva cuantitativa, sin embargo, en ocasiones no es suficiente para comprenderla en su totalidad, es por ello que resulta interesante abordarla a partir de la metodología cualitativa, sin necesidad de atarse a determinadas áreas y preguntas específicas, sino entender cómo a partir de una estructura válida, podemos

\footnotetext{
${ }^{1}$ Estudiante de la Maestría en Psicología con Orientación en Calidad de Vida y Salud, CUSUR, Universidad de Guadalajara. Correo electrónico: psi.janethtorres@gmail.com
} 
encontrar información relevante y emergente para la población de estudio. De acuerdo con Tonon (2014) "los métodos cualitativos buscan sus problemas en la vida diaria, se utilizan para estudiar significados intersubjetivos y localizados, y están orientados al descubrimiento, no a la verificación" (p. 5). Por lo tanto, el objetivo de este ensayo es conocer aspectos relevantes de la metodología cualitativa como herramienta para comprender la calidad de vida de las personas.

\section{Calidad de vida}

Seth (1889) fue probablemente el primero en conceptualizar el bienestar social como un estándar ético, haciendo énfasis en la calidad, no obstante, el interés por la CV, surge en Estados Unidos tras la Segunda Guerra Mundial debido a la inseguridad financiera, por la que se evaluaban datos objetivos como nivel socioeconómico, educación y vivienda (Urzúa \& Caqueo-Urízar, 2012). A partir de entonces se crearon definiciones centradas en las condiciones de vida, aquellas relacionadas con mediciones observables, las cuales bien podrían medirse a lo largo del tiempo o la vida, y obtener un valor cuantitativo (Hidalgo, 2008). En consecuencia, se crearon definiciones que se centran en la subjetividad, a lo que se le nombra satisfacción con la vida; en este sentido, se estaría midiendo la vida en términos de calidad, al considerar lo que se vive y la forma en que se vive (Hidalgo, 2008). No obstante, tanto las definiciones centradas en las condiciones de vida como en la satisfacción con la vida, tienen sus pros y contras, pues si bien las condiciones explican muy poco, las centradas en la satisfacción pueden alejarse de la realidad. Por ende, se deben considerar ambas partes para una mayor comprensión; desde esta perspectiva, la CV es un constructo complejo y multidimensional, su estudio depende del tiempo y de las características de la persona como un ser bio-psico-social (Hidalgo, 2008; Sinha, 2019).

Uno de los modelos iniciales de esta perspectiva es el de Smith et al. (1999), que proponen que la percepción de la calidad de vida tiene como base los procesos cognitivos para formular actitudes y juicios, la persona debe identificar los dominios relevantes que la componen para determinar los estándares en cada uno de los dominios, así como integrar los juicios de cada uno de estos en una evaluación general. Otros autores como Michalos (1985) comparten la idea de que la CV surge de las discrepancias, por ejemplo, entre lo que se tiene y se quiere, lo mejor del pasado y expectativas, lo que se merece y lo que se necesita. Estas perspectivas cognitivas, surgen de la teoría de Festinger (1954) sobre la comparación social, que hace referencia a las comparaciones que realiza una persona de sus opiniones y habilidades en relación a las de su grupo, ya que existe una tendencia biológica de evaluar, con el fin de responder de forma apegada a las expectativas y normas sociales, al compararse 
con mejores o peores situaciones, permitiendo la influencia sobre el estado anímico, el afrontamiento, la motivación o la satisfacción personal.

\section{Metodología cualitativa}

Generalmente la calidad de vida se evalúa mediante cuestionarios psicométricos pero es posible valorarla a través de métodos cualitativos. La metodología cualitativa es un proceso holístico, inductivo e interactivo que requiere un trabajo riguroso con el propósito de comprender el significado de los eventos, situaciones y acciones de los participantes para identificar y generar teoría; el proceso comienza con el reconocimiento del investigador de su posición sociocultural e histórica, quien se encuentra personalmente involucrado y debe ser versátil, tener amplio conocimiento de la teoría, ser persistente, centrado y capaz de conceptualizar, escribir y comunicar (Tonon, 2014). Sus ejes son el descubrimiento y la comprensión, por lo que requiere ir más allá del conocimiento, al comprender un contexto que se construye mediante conceptos, variables, relaciones, símbolos, reglas, creencias y teorías de personas o grupos, e implica aspectos ontológicos, epistemológicos, metodológicos y axiológicos para comprender, construir o revelar el significado contextual y hacer observable el mundo de los participantes (Tonon, 2015).

Las muestras en metodología cualitativa pueden evolucionar en función de los objetivos, las metas del proyecto, la teoría y los conceptos. La recopilación de datos implica considerar los sentimientos y emociones involucrados y construidos entre los participantes, estimula la colaboración y permite reducir la incertidumbre; la información se codifica y categoriza en función de cómo se construye el significado y se relaciona con las normas, valores, costumbres, ideología y prácticas socioculturales, por lo que la interpretación y la capacidad para presentar argumentación válida es muy importante, mientras, la validez es relativa, depende de los propósitos y circunstancias e implica un cuestionamiento teórico de lo ocurrido en la investigación (Tonon, 2014). La descripción de los datos puede ser fina, es decir, sin mostrar intenciones, motivos, significados o circunstancias, o puede ser densa al descubrir e interpretar el conocimiento o las estructuras de relaciones de los participantes para construir una teoría, pero este es un proceso largo y profundo que implica formular ideas en un esquema lógico, sistemático y explicativo, por lo tanto, la metodología cualitativa ayudaría a comprender las experiencias de bienestar de las personas de manera profunda y personalizada (Tonon, 2015).

\section{Conclusiones}


En definitiva, el constructo calidad de vida es sumamente complejo, ya que es multidimensional y depende del contexto y del tiempo en gran medida. Se ha definido como una variable matemática u observable, o bien, puramente subjetiva, así como de ambas formas, pero se ha medido principalmente mediante metodología cuantitativa. Sin embargo, este ensayo sostiene la idea de que conocer la CV a través de la metodología cualitativa tiene beneficios.

Existen diversos métodos cualitativos como teoría fundamentada, fenomenología, hermenéutica, etnografía, análisis temático, análisis del discurso y métodos participativos, pero no importa cuál de estos se elija, ya que esta elección dependerá de las características contempladas; el elegir este tipo de metodología implica tomar cierta decisión filosófica, teórica y política que es fundamental para la comprensión de las experiencias de bienestar de las personas y para el descubrimiento de nuevos temas relacionados con el campo de la $\mathrm{CV}$, ya que permite considerar las percepciones, opiniones, sentimientos, ideas e interpretaciones de significados de las personas dentro de su contexto social y cultural, así como trabajar con las potencialidades de los participantes desde un enfoque comunitario sociopolítico, basado en los derechos humanos, en el que se desenvuelve una persona o un grupo como parte de su realidad social (Tonon, 2015). El hecho es que la metodología cualitativa está demostrando ser una herramienta eficiente para comprender la perspectiva o las experiencias de bienestar de las personas, ya que cuenta con técnicas enriquecedoras y permite comprender información valiosa y no predeterminada acerca de los participantes, por ende, se concluye que la metodología cualitativa es relevante y eficaz para el estudio de la CV.

La autora agradece al CONACyT por la beca otorgada durante los estudios de maestría.

\section{Referencias bibliográficas}

Festinger, L. (1954). A Theory of Social Comparison Processes. Human Relations, 7(2), 117140. doi.org/10.1177/001872675400700202

Hidalgo, C. (2008). De los comportamientos de riesgo a la calidad de vida de los adolescentes. Conceptos, instrumentos y estudios. Prometeo editores.

Michalos, A. (1985). Multiple discrepancies theory. Social Indicators Research, 16, 347-413.

Seth, J. (1889). The evolution of morality. In Mind. Oxford University Press on behalf of the Mind Association, 14(53). 
Sinha, B. (2019). Introduction: An Overview of the Concept of Quality of Life. En B. Sinha (Eds.), Multidimensional Approach to Quality of Life Issues (pp. 3-23). Springer.

Smith, K., Avis, N. \& Assman, S. (1999). Distinguishing between quality of life and health status in quality of life research: A meta-analysis. Quality of life research, 8, 447459.

Tonon, G. (2015) Relevance of the use of qualitative methods for the study of quality of life. En G. Tonon (Eds.), Qualitative Studies in Quality of Life Methodology and Practice. Social Indicators Research Series (pp. 3-21). Springer.

Tonon, G. (2014) Qualitative Methods. En A. C. Michalos (Eds.), Encyclopedia of Quality of Life and Well-Being Research (pp. 522-525). Springer.

Urzúa, A. y Caqueo-Urízar, A. (2012). Calidad de vida: Una revisión teórica del concepto. Terapia Psicológica, 30(1), 61-71. doi.org/10.4067/S0718-48082012000100006 